\title{
Performance of the New Turbine Mid-Level Critical Care Ventilators
}

\author{
Carlos Delgado MD, Jose E Romero, Jaume Puig MD PhD, Ana Izquierdo MD, \\ Carlos Ferrando MD PhD, F Javier Belda MD PhD, and Marina Soro MD PhD
}

\begin{abstract}
BACKGROUND: During recent years, ventilators using turbines as flow-generating systems have become increasingly more relevant. This bench study was designed to compare triggering and pressurization of 7 turbine mid-level ICU ventilators. METHODS: We used a dual-chamber lung model to test 7 mid-level ICU ventilators in pressure support mode with levels of 10,15 , and $20 \mathrm{~cm} \mathrm{H}_{2} \mathrm{O}$ with 2 PEEP levels of $5 \mathrm{~cm} \mathrm{H}_{2} \mathrm{O}$ and the minimum level allowed by the ventilator. A ventilator was connected to the master chamber to simulate 2 different effort levels. Pressure drop, trigger delay time, time to minimum pressure, and pressure time products (PTP) during trigger and the first 300 and 500 ms were analyzed. RESULTS: In the trigger evaluation, the Savina had the highest delay time, whereas the C2, the V60, and the Trilogy had the lowest pressure drops and PTP values in both effort levels. In pressurization capacity assessment using ideal PTP300 and PTP500 percentages, the $\mathrm{C} 2$ and the V680 had the best results, and the Carina and the Savina had lower values, with no differences between both effort levels. Differences between PEEP levels did not seem to be relevant. CONCLUSIONS: Pressure support mode for tested ventilators worked properly, but pressurization capacity and trigger function performance were clearly superior in the newest machines. The use of PEEP did not modify the results. Key words: ventilators; mechanical ventilation; critical care; models; equipment design; bench study. [Respir Care 2017;62(1):34-41. (C) 2017 Daedalus Enterprises]
\end{abstract}

\section{Introduction}

In recent years, several new mid-level critical care ventilators have been launched in the marketplace. Some of them include new technologies to help improve adaptation to the different ventilatory situations, mainly in terms of synchronism and pressurization capacity. Both aspects have been proved essential to optimize the patient's work of breathing. ${ }^{1-6}$

Mechanical ventilators feature different systems for inspiratory flow generation (pneumatic bellows and pistons,

Drs Delgado, Puig, Izquierdo, Ferrando, Belda, and Soro are affiliated with the Department of Anesthesia and Critical Care, Hospital Clínico Universitario, Valencia, Spain. Mr Romero is affiliated with IBIME, ITACA Center, Universidad Politécnica de Valencia, Valencia, Spain.

The authors have disclosed no conflicts of interest.

Correspondence: Dr Carlos Delgado Navarro, Department of Anesthesia and Critical Care, Hospital Clínico Universitario de Valencia, Blasco Ibáñez 17, 46010, Valencia, Spain. E-mail: cardelna@gmail.com.

DOI: $10.4187 /$ respcare. 04938 solenoid valves, and scissor valves), but there is currently a trend toward installing turbines, which are rotors that proportionally accelerate flow at high speed. ${ }^{7}$ At end expiration, turbines are still in movement and a constant flow is generated, creating a low level of PEEP/CPAP, which usually is the lowest preset value. ${ }^{8}$

Several bench studies using physical lung models have been published, and they have proved useful for highlighting technical differences. These differences are probably the cause of the heterogeneous clinical performance of ventilators observed in practice. ${ }^{9-12}$ With this methodology, several different generation ventilators have been compared. These comparisons have shown that ventilator designs throughout the last 20 years have significantly improved, in terms of synchronization and pressurization. ${ }^{13}$ Pressure support is the default mode generally used to assess performance, because its use is widespread and it has been proven to benefit patient synchronization. ${ }^{14-16}$

When using this methodology for comparing ventilators designed with either turbines or pneumatic compression valves, older turbine-based ventilators perform slightly less well than the most recent compressed-gas ventilators; however, the newer machines have closer levels of perfor- 
mance, with turbine-based ventilators providing slightly better trigger function and pressurization capacities. ${ }^{17}$

When comparing ventilators with the same flow generation technology, even though similar behaviors would be expected, there are noticeable differences in clinical practice, and they can be quite significant, especially when they impact adaptation to patients' needs. Several bench studies have compared some ventilators designed for disparate purposes, lacking turbine technology, and they have demonstrated that the newest ones tend to perform better. ${ }^{18,19}$ However, there are no comparative studies using the most recent turbine ventilators in the critical care environment.

The purpose of this study was to analyze the performance of 7 mid-level ICU turbine ventilators currently available, comparing them to show the differences in their performance using pressure support ventilation (PSV) mode, with a strict and systematic bench protocol using a physical lung model.

\section{Methods}

The turbine ventilators selected were $\mathrm{C} 2$ (Hamilton, Bonaduz, Switzerland), Vela (CareFusion, San Diego, California), Savina (Dräger, Lubeck, Germany), Carina (Dräger), V60 (Respironics, Philips, Carlsbad, California), Trilogy (Respironics), and V680 (Respironics). Differential characteristics of all of them are summarized in Table 1. Experiments were run using the default circuit provided by the manufacturer with the ventilator in each case.

\section{Physical Lung Model}

Analysis was carried out using a physical bi-compartmental validated lung model (Dual Adult PneuView 5600i, Michigan Instruments, Grand Rapids, Michigan), previously used in several similar bench studies. ${ }^{8}{ }^{14-20}$ The model consists of 2 separate chambers, connected by a rigid metal bar. One of the chambers was connected to an ICU ventilator functioning as a primary lung (Evita 4, Dräger, Lubeck, Germany), so that positive pressure insufflated in the first chamber would produce a negative pressure in the second chamber, "patient chamber" or secondary lung, which is detected as an inspiratory effort by the tested ventilator, which is connected to the secondary lung. Volume control mode was set in the master ventilator. Changes in the master ventilator were performed to modify effort's magnitude and duration.

Compliance and resistance of each chamber were separately adjusted. Compliance was set using the mechanism featured by the lung model itself, by sliding a strain spring by the aid of a precision adjustment scale. A specifically designed calibrated linear resistance was used, rather than the default fixed orifice resistances provided with the lung

\section{QUICK LOOK}

\section{Current knowledge}

New flow-generating systems for mid-level ICU ventilators, such as turbines, have been introduced. Bench studies have shown different performances in pressurization and trigger capacities among ventilators using other technologies, but turbine ventilators have not been compared so far.

\section{What this paper contributes to our knowledge}

Using a physical lung model, currently available turbine mid-level ICU ventilators worked properly. However, under our experimental conditions, the newest machines clearly had the best results: the $\mathrm{C} 2$, the V680, and the Trilogy (AC) had higher pressurization capacity, and the $\mathrm{C} 2$, the V60, and the Trilogy (AC) had better trigger performance. The use of PEEP did not affect these features in the tested ventilators.

model; thus, any other resistive elements such as endotracheal tubes were avoided in the simulation. During all tests, compliance and resistance in the primary and the secondary ventilator were adjusted with normal values of compliance of the respiratory system $=50 \mathrm{~mL} / \mathrm{cm} \mathrm{H}_{2} \mathrm{O}$ and resistance $=5 \mathrm{~cm} \mathrm{H}_{2} \mathrm{O} / \mathrm{L} / \mathrm{s}$.

All tested ventilators were configured in PSV mode with 10,15 , and $20 \mathrm{~cm} \mathrm{H}_{2} \mathrm{O}$. PEEP value was adjusted to the minimum allowed by the machine (zero PEEP) and $5 \mathrm{~cm} \mathrm{H}_{2} \mathrm{O}$. Trigger sensitivity was adjusted to the highest sensitivity allowed by the ventilator without creating autotriggering. Pressurization slope was adjusted to the steepest allowed.

The primary ventilator was configured to simulate 2 inspiratory effort levels: high and low. The intensity of the effort was quantified using the pressure drop in $0.1 \mathrm{~s}$ during an occlusion maneuver $\left(\mathrm{P}_{0.1}\right)$ created at the exit of the secondary lung chamber. A 12-cycle per min breathing frequency was used, as well as a 1-s inspiratory time, and 2 constant inspiratory flow levels: $0.24 \mathrm{~L} / \mathrm{s}$ and $0.44 \mathrm{~L} / \mathrm{s}$, to obtain $\mathrm{P}_{0.1}$ values of $2 \mathrm{~cm} \mathrm{H}_{2} \mathrm{O}$ (low effort) and $4 \mathrm{~cm} \mathrm{H}_{2} \mathrm{O}$ (high effort).

\section{Measuring System}

Flow measurements were obtained with a Fleish No.2 pneumotachograph, inserted in between the secondary lung and the tested ventilator. A differential pressure transducer was used (Validyne MP45, $\pm 2.5 \mathrm{~cm} \mathrm{H}_{2} \mathrm{O}$, Northridge, California), and the signal was integrated to obtain volume. Airway pressure was measured in the distal extreme 


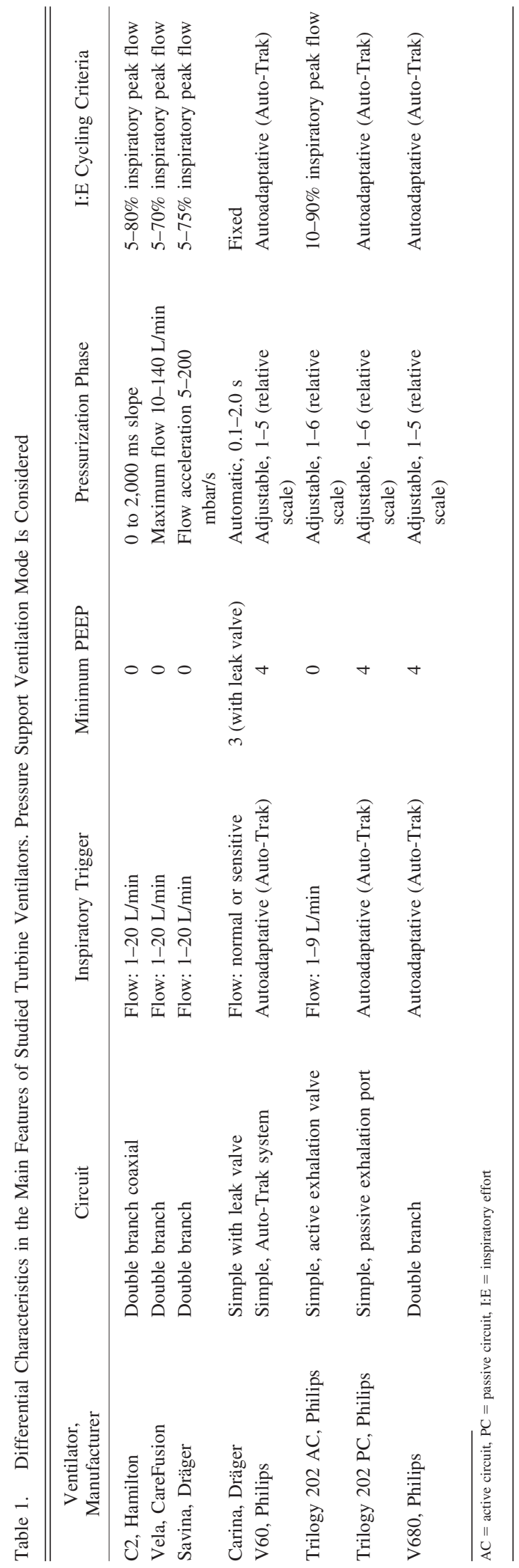

of the circuit (Validyne MP45, $\pm 80 \mathrm{~cm} \mathrm{H}_{2} \mathrm{O}$ ). The pneumotachograph was calibrated by measuring the volume derived from the flow signal using a 1-L standard syringe (Harvard Apparatus, Holliston, Massachusetts). Pressure transducers were calibrated to $20 \mathrm{~cm} \mathrm{H}_{2} \mathrm{O}$ using a water manometer (HG15, General Tools, Secaucus, New Jersey).

Signals were obtained in real time with an analogdigital converter (MP100; Biopac Systems, Goleta, California), using a 200-Hz sampling frequency, and they were stored in a laptop for revision with specific software (AcqKnowledge software, Biopac Systems). Signal registration was performed for every configuration after reaching a steady state in the system for $60 \mathrm{~s}$.

\section{Measurements}

Triggering. To evaluate trigger quality, flow sensitivity was adjusted to the lowest level allowed by every ventilator, or $0.5 \mathrm{~L} / \mathrm{min}$ by default. The following parameters were measured:

- Airway Pressure Drop: Represents the maximum pressure drop in the airway, measured from the baseline, in $\mathrm{cm}_{2} \mathrm{O}$. Pressure drop reflects the amount of inspiratory effort required to trigger a new cycle in pressure support ventilation mode; the lower its value, the less work is required.

- Time to Pressure Minimum: Represents the time since the beginning of the inspiratory effort (identified as the point where flow steeply increases and pressure drops) until the moment of reaching the lowest airway pressure, measured in ms. This value represents the ventilator response time; the shorter it is, the faster this response is.

- Trigger Delay Time: Represents the time since the beginning of the inspiratory effort until the beginning of inspiratory flow, which is the moment when pressure reaches PEEP value again. It represents the effective triggering time, since supportive pressure is only effective from that moment on.

- Pressure Time Product (PTP): Represents the area under the pressure curve from the beginning of inspiration until airway pressure reaches PEEP value again. It represents the effort needed until the assistance is effective; therefore, the lower it is, the lower the effort needed from the patient.

Pressurization capacity. The following parameters were measured:

- Ideal PTP300 percentage (iPTP300\%): PTP300 is the area under the pressure curve during the first $300 \mathrm{~ms}$ of a cycle. $\mathrm{PPTP} 300 \%$ is the fraction represented by the actual PTP300 value out of the ideal area for PTP300. The use of percentages gives a better image of its sig- 


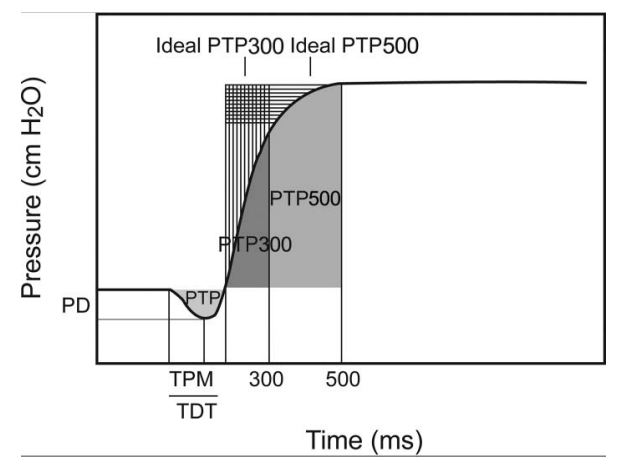

Fig. 1. Measurements performed in every cycle. $P D=$ pressure drop, TPM = time to pressure minimum, TDT = trigger delay time, PTP $=$ pressure time product during trigger, PTP $300=$ PTP during the first $300 \mathrm{~ms}$, PTP500: PTP during the first $500 \mathrm{~ms}$.

nificance and has been previously used in similar studies. $^{20}$

- Ideal PTP500 percentage (iPTP500\%): Same concept as iPTP300\% but calculated during the first $500 \mathrm{~ms}$ instead.

A theoretical representation of the measurements in a model cycle can be found in Figure 1.

\section{Processing and Statistics}

To analyze every cycle individually, a specifically designed script for the MATLAB system (Mathworks, Natick, Massachusetts) was used. Results obtained for every parameter are expressed as means and interquartile ranges of the values extracted from 5 cycles for every configuration.

Statistical analysis was performed using SPSS20.0 (SPSS, Chicago, Illinois) and Microsoft Excel (Microsoft, Redmond, Washington). All results are expressed as mean \pm SD or median with $95 \% \mathrm{CI}$, depending on the normal or non-normal distribution of the variables. Comparative statistics were done using the Kruskal-Wallis test for variance analysis. Post hoc analysis was performed with the Dunn test. Statistical significance was adjusted to a $P$ value $<.05$, and differences $>10 \%$ were considered relevant.

\section{Results}

Ninety-six different conditions were tested. No failures in trigger or premature cycles were experienced with any of the ventilators.

\section{Trigger Evaluation}

Pressure drop, time to pressure drop, trigger delay time, and PTP measurements in all experimental conditions are shown in Figures 2 and 3.
Pressure drop and PTP were significantly lower in the $\mathrm{C} 2$, the Trilogy (using active circuit), and the V60, which are among the newest ventilators. PTP and time to pressure drop found in the tests with the Savina were significantly higher compared with those in the rest of the ventilators (Figs. 2 and 3).

In general, no differences were found in the comparisons between minimum PEEP and a PEEP of $5 \mathrm{~cm} \mathrm{H}_{2} \mathrm{O}$. We only found a significantly higher pressure drop with a PEEP of $5 \mathrm{~cm} \mathrm{H}_{2} \mathrm{O}$ with the Vela ventilator with the higher effort condition $\left(\mathrm{P}_{0.1}\right.$ of $\left.4 \mathrm{~cm} \mathrm{H}_{2} \mathrm{O}\right)$. Also with the same effort and PEEP conditions, significantly lower values of pressure drop were found with the V680, the Trilogy (with active circuit), and the V60 (Figs. 2 and 3).

\section{Pressurization Capacity Evaluation}

iPTP300\% and iPTP500\% measurements in all experimental conditions are shown in Figures 4 and 5. iPTP300\% and iPTP500\% were significantly higher in the $\mathrm{C} 2$ and the V680 compared with the other ventilators, for both levels of effort. Conversely, the Carina and the Savina had significantly lower iPTP300\% and iPTP500\% values compared with the other ventilators, also for both levels of effort (Figs. 4 and 5). Significant differences were only found in iPTP300\% and iPTP500\% with different levels of PEEP for the V680, with both $\mathrm{P}_{0.1}$ levels (Figs. 4 and 5).

\section{Discussion}

To our knowledge, this is the first study where the performances of the currently available mid-level ICU turbine ventilators have been assessed during PSV applying a strict bench protocol with a physical lung model. Major findings can be summarized as follows: (1) pressure support mode for all 7 tested ventilators works properly; (2) trigger function performance is clearly superior in the newest ventilators, while some of the oldest ones have poorer results; (3) performance in terms of pressurization capacity is higher in the devices with the newest turbines, compared with older ventilators; (4) PEEP does not seem to affect trigger and pressurization capacity, even though some differences can be observed in some machines, with doubtful significance.

In this study, trigger delays were observed close to 100 $\mathrm{ms}$ in the newest ventilators (the $\mathrm{C} 2$, the V680, the Trilogy, the V60, and the Vela), whereas in some of the older ones trigger delays were close to $200 \mathrm{~ms}$ (the Carina and the Savina), with either high or low effort. Trigger delay time and time to pressure minimum had concordant results. Effort level did affect trigger PTP and pressure drop in all the machines, in accordance with findings in similar studies. ${ }^{9}$ 

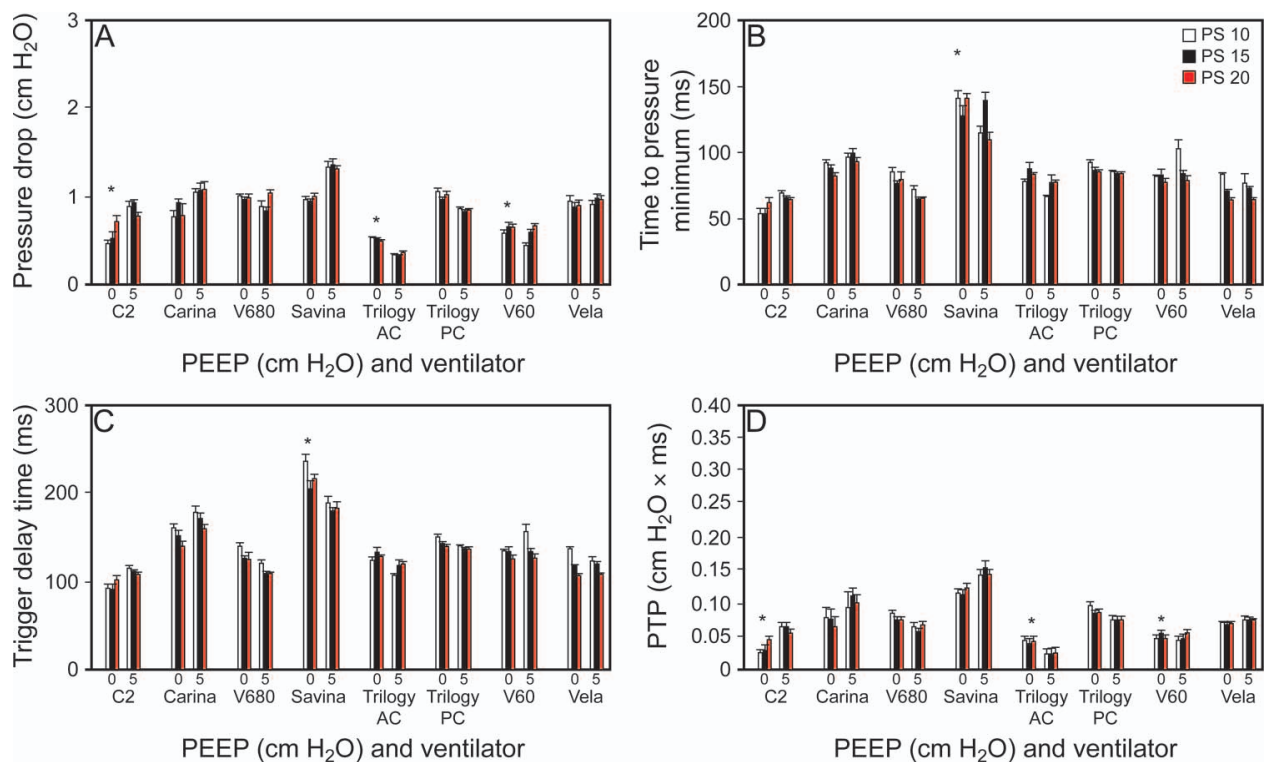

Fig. 2. Trigger evaluation results, evaluated with pressure drop (A), time to pressure minimum (B), trigger delay time $(C)$, and pressure-time product $(P T P)(D)$ with a low inspiratory effort level $\left(P 0.12 \mathrm{~cm} \mathrm{H} \mathrm{H}_{2} \mathrm{O}\right)$ with minimum allowed PEEP value according to each machine $(0 \mathrm{~cm}$ $\mathrm{H}_{2} \mathrm{O}$ ) and $5 \mathrm{~cm} \mathrm{H}_{2} \mathrm{O}(5)$ for 3 levels of pressure support (PS) ventilation of 10, 15, and $20 \mathrm{~cm} \mathrm{H}_{2} \mathrm{O}$. * $P<.05$ between ventilators.
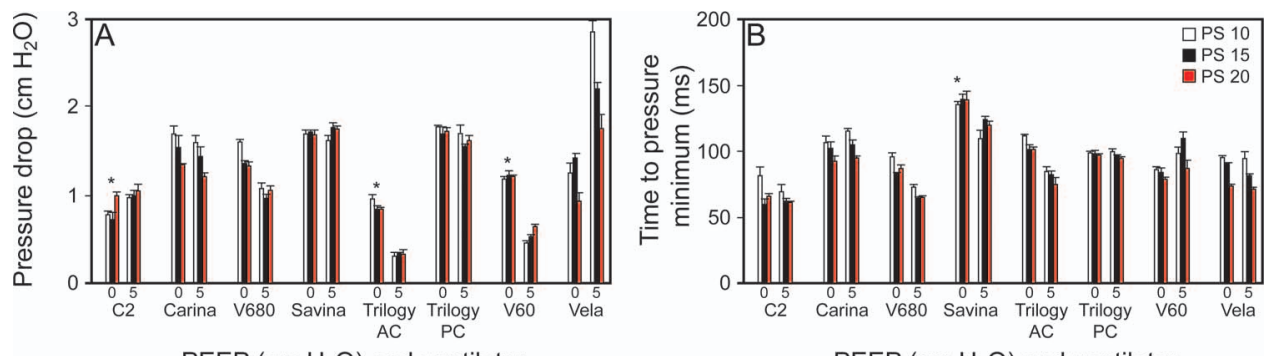

PEEP $\left(\mathrm{cm} \mathrm{H}_{2} \mathrm{O}\right)$ and ventilator

PEEP $\left(\mathrm{cm} \mathrm{H}_{2} \mathrm{O}\right)$ and ventilator
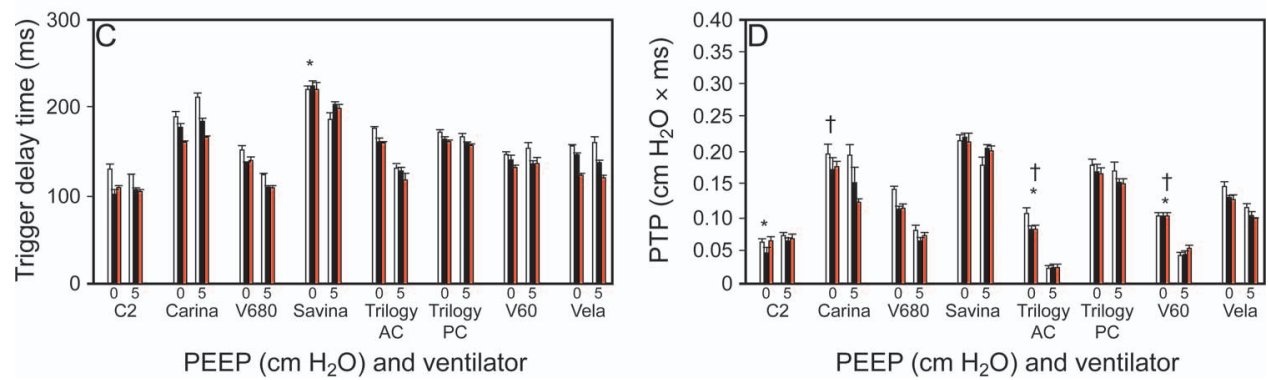

Fig. 3. Trigger evaluation results, evaluated with parameters pressure drop, time to pressure minimum, trigger delay time, and PTP (pressure time product) with a high inspiratory effort level $\left(\mathrm{PO} .14 \mathrm{~cm} \mathrm{H} \mathrm{H}_{2} \mathrm{O}\right)$ with minimum allowed PEEP value according to each machine $\left(0 \mathrm{~cm} \mathrm{H}_{2} \mathrm{O}\right)$ and $5 \mathrm{~cm}$ $\mathrm{H}_{2} \mathrm{O}(5)$ for 3 levels of pressure support (PS) ventilation of 10,15 , and $20 \mathrm{~cm} \mathrm{H}_{2} \mathrm{O}$. * Significant differences comparing ventilators $(P<.05)$. † Significant differences comparing minimum PEEP and PEEP $5 \mathrm{~cm} \mathrm{H}_{2} \mathrm{O}(P<.05)$.

We found significantly higher values for trigger delay time in the Savina ventilator compared with findings in a similar study by Thille et al featuring this machine, ${ }^{17}$ but other ventilators included in this study had concordant results. This last study used different $\mathrm{P}_{0.1}$ levels, and results were expressed as means of 4 different effort intensities overall.
Our results are in line with those found in similar studies by Tassaux et $\mathrm{al}^{9}$ and Battisti et al, ${ }^{10}$ where pressure drop and PTP were also evaluated, even though different ventilators were tested. In contrast to our results, inspiratory work load required to trigger ventilation represented by PTP and secondarily by pressure drop is higher overall. These differences can probably be explained first because 

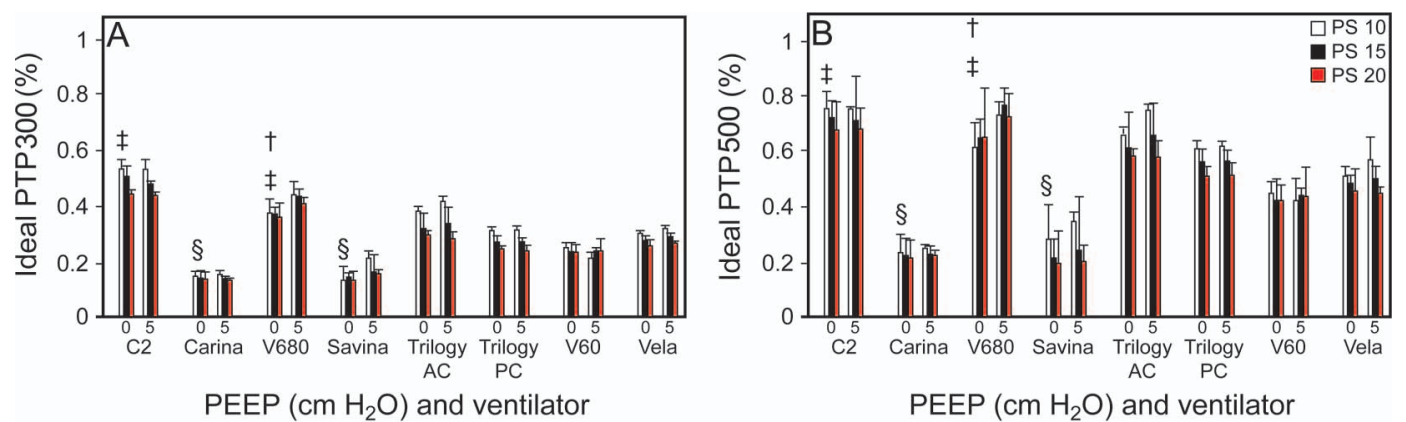

Fig. 4. Pressurization capacity results, assessed with parameters ideal PTP300\% and ideal PTP500\% with a low inspiration effort level (P0.1 $2 \mathrm{~cm} \mathrm{H}_{2} \mathrm{O}$ ) with minimum PEEP value allowed by each ventilator $\left(0 \mathrm{~cm} \mathrm{H}_{2} \mathrm{O}\right)$ and $5 \mathrm{~cm} \mathrm{H}_{2} \mathrm{O}$ (5) for 3 levels of pressure support (PS) ventilation of 10,15 , and $20 \mathrm{~cm} \mathrm{H}_{2} \mathrm{O}$. $\ddagger$ Ventilators with significantly higher values $(P<.05)$. $\S$ Ventilators with significantly lower values $(P<.05)$. † Significant differences comparing minimum PEEP and PEEP $5 \mathrm{~cm} \mathrm{H}_{2} \mathrm{O}(P<.05)$.
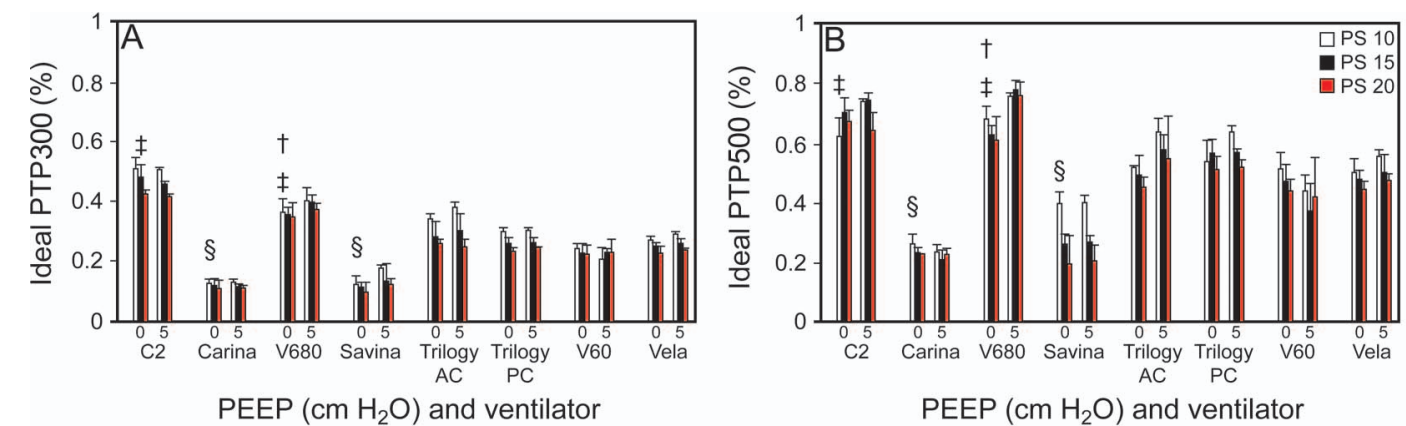

Fig. 5. Pressurization capacity results, assessed with parameter ideal PTP $300 \%$ and ideal PTP500\% with a high inspiration effort level (P0.1 $\left.4 \mathrm{~cm} \mathrm{H}_{2} \mathrm{O}\right)$ with minimum PEEP value allowed by each ventilator $\left(0 \mathrm{~cm} \mathrm{H}_{2} \mathrm{O}\right)$ and $5 \mathrm{~cm} \mathrm{H}_{2} \mathrm{O}(5)$ for 3 levels of pressure support (PS) ventilation of 10,15 , and $20 \mathrm{~cm} \mathrm{H} \mathrm{H}_{2} \mathrm{O}$. $¥$ Ventilators with significantly higher values $(P<.05)$. $\S$ Ventilators with significantly lower values $(P<.05)$. † Significant differences comparing minimum PEEP and PEEP $5 \mathrm{~cm} \mathrm{H}_{2} \mathrm{O}(P<.05)$.

different machines were tested in these 2 studies. Second, $\mathrm{P}_{0.1}$ parameter was not used to set up the inspiratory effort; the amount of pressure set in the master ventilator to mimic inspiratory effort was used instead. In fact, another study by Jaber et al, ${ }^{18}$ using $\mathrm{P}_{0.1}$ parameter did show PTP values similar to our findings, even though different ventilators were tested as well.

The lowest PTP values were obtained with the most modern ventilators, especially the $\mathrm{C} 2$, the $\mathrm{V} 60$, and the Trilogy (AC). In agreement with previous data, the oldest ventilators obtained the highest values (the Carina and the Savina).

In the assessment of pressurization capacity using iPTP300\% and iPTP500\%, data indicate that the newest turbines (the $\mathrm{C} 2$, the V680, and the Trilogy $\mathrm{AC}$ ) have a better pressurization capacity, as shown in our results (Figs. 4 and 5), compared with the older ones (the Carina and the Savina), independently of the ideal PTP analyzed. Testing 2 different levels of effort did not affect these differences. Our results are in agreement with results from the only study we could find that tested a turbine machine included in our protocol; this study was by Vignaux et al, ${ }^{20}$ where a very similar iPTP500\% was found for the Vela ventilator.
Only the Carina and the Savina did not reach a $25 \%$ iPTP300\%, whereas the C2, the V680, and the Trilogy AC had the highest values. In the case of iPTP500\%, only the $\mathrm{C} 2$ and the V680 reached a $60 \%$ value, and again, the Carina and the Savina had the lowest values.

This study was performed using a physical lung model instead of real patients, which is a clear limitation, since performance in humans is not comparable to the results obtained through physical simulation. However, this methodology allows standardization and reproducibility of the mechanical conditions under which experiments are performed, which allows testing of all the ventilators under very similar circumstances, which is highly advantageous.

Our dual chamber lung model (Dual Adult PnewView 5600i, Michigan Instruments, Grand Rapids, Michigan) was used in most of the studies we reviewed, $9,10,13,17,18,20$ as well as data acquisition settings. Among these studies, a master ventilator was set in volume control mode in only one. ${ }^{18}$ In the other research, pressure control mode was used to mimic patient's effort, as in the report by Thille et al. ${ }^{17}$ In this study, the aforementioned longer trigger delay times in the Savina ventilator in comparison with 


\section{Performance of Turbine Critical Care Ventilators}

our findings could be related to this difference in triggering pattern.

Most of these studies used the same values of compliance and resistance that we used in our protocol (compliance $50 \mathrm{~mL} / \mathrm{cmH}_{2} \mathrm{O}$ and resistance $5 \mathrm{cmH}_{2} \mathrm{O} / \mathrm{L} / \mathrm{s}$ ). In the study by Thille et al, ${ }^{17}$ compliance $100 \mathrm{~mL} / \mathrm{cmH}_{2} \mathrm{O}$ was set in the lung model. In the protocol by Richard et al, ${ }^{13}$ compliance $80 \mathrm{~mL} / \mathrm{cmH}_{2} \mathrm{O}$ was used, and an 8.0 ETT was connected to the test chamber to create a resistance of 7 $\mathrm{cmH}_{2} \mathrm{O} / \mathrm{L} / \mathrm{s}$.

Different lung model designs were used in some of the reviewed studies. Bunburaphong et ${ }^{11}$ al used a bellows-in-a-box lung model with negative pressure and pleural space simulation. This design provides a more realistic and a narrower control of effort generation, but its setup is much more complex. A different design is also described in the study by Ferreira et al, ${ }^{12}$ where a computerized mechanical lung simulator is used, consisting of a piston moving inside a cylinder according to an equation of motion. Unlike dual chamber models, this lung simulator does not simulate expiratory efforts, but findings in trigger delay time and pressure drop in Savina and Vela ventilators in this study were very similar to our results.

Regarding the fact that PEEP does not seem to affect triggering or pressurization capacity, it must be taken into consideration that only 2 relatively low levels of PEEP, $\leq 5 \mathrm{~cm} \mathrm{H}_{2} \mathrm{O}$, were evaluated, whereas some patients require higher PEEP levels. Our testing at low levels of PEEP may not be adequate to know how a higher PEEP may affect triggering or pressurization performance, so conclusions should be cautiously made.

We used the V60 ventilator in an invasive ventilation setting even though it is mainly designed to be used as a noninvasive ventilation machine. We arranged this setup for this ventilator, because it can be configured for that purpose as well; therefore, these results must be evaluated considering that they are only applicable for invasive ventilation, which is not its native design.

\section{Conclusions}

For the turbine mid-level ICU ventilators tested, pressure support ventilation functioned correctly, whereas, under our experimental conditions, the most recent turbines have the best results in terms of trigger function $(\mathrm{C} 2, \mathrm{~V} 60$, Trilogy AC) and pressurization capacity $(\mathrm{C} 2$, V680, Trilogy AC) compared with the older models. The use of PEEP did not modify trigger or pressurization results.

Extrapolations of the findings in this study must be cautiously done. Clinical studies assessing these features should be performed to check whether these results actu- ally do comply with the findings obtained in patients undergoing mechanical ventilation in the ICU.

\section{REFERENCES}

1. Chiumello D, Pelosi P, Croci M, Bigatello L, Gattinoni L. The effects of pressurization rate on breathing pattern, work of breathing, gas exchange and patient comfort in pressure support ventilation. Eur Respir J 2001;18:107-114.

2. Aslanian P, El Atrous S, Isabey D, Valente E, Corsi D, Harf A et al. Effects of flow triggering on breathing effort during partial ventilatory support. Am J Respir Crit Care Med 1998;157(1):135-143.

3. Brochard L, Mancebo J, Amaro P, Mollo JL, Lorino H, Lemaire F. Comparison of the effects of pressure support ventilation delivered by three different ventilators during weaning from mechanical ventilation. Intensive Care Med 1995;21(11):913-919.

4. Sassoon CS, Del Rosario N, Fei R, Rheeman CH, Gruer SE, Mahutte $\mathrm{CK}$, Kees $\mathrm{C}$. Influence of pressure- and flow-triggered synchronous intermittent mandatory ventilation on inspiratory muscle work. Crit Care Med 1994;22(12):1933-1941.

5. Bonmarchand G, Chevron V, Menard J-F, Girault C, Moritz-Berthelot F, Pasquis P, Leroy J. Effects of pressure ramp slope values on the work of breathing during pressure support ventilation in restrictive patients. Crit Care Med 1999;27(4):715-722.

6. Bonmarchand G, Chevron V, Jusserand D, Girault F, Moritz Leroy $\mathrm{P}$ et al. Increased initial flow reduces inspiratory work of breathing during pressure support ventilation in patients with exacerbation of chronic obstructive pulmonary disease. Intensive Care Med 1996; 22(11):1147-1154.

7. Zhou YX, Hu ZY, Zhan M, Ge B, Xie HM. Design of a noninvasive ventilator's turbine. Zhongguo Yi Liao Qi Xie Za Zhi 2008;32(2): 97-99.

8. Pestaña D. Positive airway pressure: CPAP and PEEP. In: Belda FJ, Lloréns J, eds. Mechanical ventilation in anesthesia and critical care. 4th ed. Madrid, Spain: Arán;2009:973-995.

9. Tassaux D, Strasser S, Fonseca S, Dalmas E, Jolliet P. Comparative bench study of triggering, pressurization, and cycling between the home ventilator VPAP II and three ICU ventilators. Intensive Care Med 2002;28(9):1254-1261.

10. Battisti A, Tassaux D, Janssens JP, Michotte JB, Jaber S, Jolliet P. Performance characteristics of 10 home mechanical ventilators in pressure-support mode: a comparative bench study. Chest 2005; 127(5):1784-1792.

11. Bunburaphong $\mathrm{T}$, Imanaka $\mathrm{H}$, Nishimura $\mathrm{M}$, Hess D, Kacmarek RM. Performance characteristics of bilevel pressure ventilators: a lung model study. Chest 1997;111(4):1050-1060.

12. Ferreira JC, Chipman DW, Kacmarek RM. Trigger performance of mid-level ICU mechanical ventilators during assisted ventilation: a bench study. Intensive Care Med 2008;34(9):1669-1675.

13. Richard J-C, Carlucci L, Breton N, Langlais S, Jaber S, Maggiore S et al. Bench testing of pressure support ventilation with three different generations of ventilators. Intensive Care Med 2002;28(8):1049-1057.

14. Tung A, Drum ML, Morgan S. Effect of inspiratory time on tidal volume delivery in anesthesia and intensive care unit ventilators operating in pressure control mode. J Clin Anesth 2005;17(1):8-15.

15. Christie JM, Smith RA. Pressure support ventilation decreases inspiratory work of breathing during general anesthesia and spontaneous ventilation. Anesth Analg 1992;75(2):167-171.

16. Kuhlen R, Putensen C. Maintaining spontaneous breathing efforts during mechanical ventilatory support. Intensive Care Med 1999; 25(11):1203-1205.

17. Thille AW, Lyazidi A, Richard JC, Galia F, Brochard LA. A bench study of intensive-care-unit ventilators: new versus old and turbine- 


\section{Performance of Turbine Critical Care Ventilators}

based versus compressed gas-based ventilators. Intensive Care Med 2009;35(8):1368-1376.

18. Jaber S, Tassaux D, Sebbane M, Pouzeratte Y, Battisti A, Capdevila $\mathrm{X}$ et al. Performance characteristics of five new anesthesia ventilators and four intensive care ventilators in pressure-support mode: a comparative bench study. Anesthesiology 2006;105(5):944-952.
19. Jaber S, Langlais N, Fumagalli B, Cornec S, Beydon L, Harf A, Brochard L. Performance studies of 6 new anesthesia ventilators: bench tests. Ann Fr Anesth Reanim 2000;19(1):16-22.

20. Vignaux L, Tassaux D, Jolliet P. Performance of noninvasive ventilation modes on ICU ventilators during pressure support: a bench model study. Intensive Care Med 2007;33(8):1444-1451.

This article is approved for Continuing Respiratory Care Education credit. For information and to obtain your CRCE

(free to AARC members) visit

www.rcjournal.com 